\title{
Interactive optimisation modelling using OPTIMISIR to support Melbourne water supply system operation
}

\author{
M.D.U.P. Kularathna ${ }^{a}$, B. Baker ${ }^{\text {a }}$ and S. Ennor ${ }^{\text {b }}$ \\ ${ }^{\mathrm{a}}$ Strategic Planning, Melbourne Water, Melbourne, Victoria, Australia, ${ }^{\mathrm{b}}$ Operations, Melbourne Water, \\ Melbourne, Victoria, Australia. \\ Email: udaya.kularathna@melbournewater.com.au
}

\begin{abstract}
Melbourne Water is a Victorian government owned company that provides water supply and sewerage services to Melbourne's retail water companies, and manages rivers and creeks, floodplains and the regional drainage system. The water supply system managed by Melbourne Water is a complex interconnected system of 10 storage reservoirs, over 40 service reservoirs, 160,000 hectares of catchments and hundreds of kilometres of tunnels and pipelines. The complexity of the system and the needs of stakeholders mean the way the system operates has to be based on many considerations. These considerations include: the volumes to be sourced from various sources that have differential costs, the timing of volumes sourced, the distribution of storage within the water supply system, minimising the risk of localised or system wide spills due to increased supply from various sources, minimising the risk of low storage volumes due to inadequate intakes volumes, water security, water quality, scheduled outages of system assets, operating and capital costs, and environmental factors such as healthy water ways and lower greenhouse gas emissions.
\end{abstract}

In planning for the system operation, Melbourne Water prepares an annual operating plan (AOP) that provides a forecast of anticipated system operation for the water supply system. The AOP outlines the system operations for the 12 months ahead, and identifies for each month the volumes of water to be sourced, stored, moved from one location to another, or released to waterways. The preparation of this forecast is a complex task, as the decisions on system operations need to be made under many considerations as outlined earlier. In the past, the preparation of AOP was a manual process undertaken by experienced operators assisted by decision support tools based around the calculation of water balance across the system. However, these tools did not have the capability to identify optimal operations. Instead, the tools were manually updated by experienced operators as documented in system operation guidelines, in consultation with Melbourne's retail water companies. The above approach was time consuming, often requiring up to two weeks to produce an AOP for one operating scenario.

In 2009, Melbourne Water developed in-house an optimisation modelling tool, OPTIMISIR, to assist in identifying the optimal annual operating plan. OPTIMISIR provides the flexibility for users to specify various streamflow and demand scenarios, system storage conditions, operating requirements and the aims of the optimisation in the form of an objective function. The choice two optimisation methods; Linear Programming or Quadratic Programming is also available. The output of OPTIMISIR comprises the operating decisions for each of the 12 months including: volumes to be taken from various water sources, volumes to be released to waterways, volumes to be stored in each reservoir and volumes to be transferred across the system. OPTIMISIR has two modes of use; standalone-use and the linked-use with Melbourne Water's REALM water supply system simulation model. This paper covers the standalone-use mode. With OPTIMISIR, the optimisation of 12-month operations can be completed in seconds. This enables the operators to generate at first a 'greenfield solution' that is not biased towards past operations and subsequently assess the impacts of various operating considerations by generating 'constrained solutions'.

The paper presents the OPTIMISIR modelling approach and an example application. The paper particularly highlights the decision-support rather than decision-making nature of OPTIMISIR, in which the experience of system operators and the power of an easy-to-use modelling tool are combined in identifying a detailed operating plan that meets various operating considerations and the needs of stakeholders. The paper demonstrates the suitability of OPTIMISIR, as part of a suite of optimisation and simulation modeling tools, for the operation planning of Melbourne's water supply system.

Keywords: Water supply system operation, optimisation, linear programming, OPTIMISIR, REALM 
Kularathna et al., Interactive Optimisation Modelling using OPTIMISIR to Support Melbourne Water Supply System Operation

\section{INTRODUCTION}

Operation planning of a large water supply system is a complex task undertaken based on many considerations. These considerations include: the volumes to be sourced from various sources that have differential costs, the timing of volumes sourced, the distribution of storage within the water supply system, minimising the risk of localised or system wide spills due to increased supply from various sources, minimising the risk of low storage volumes due to inadequate intakes volumes, water security, water quality, scheduled outages of system assets, operating and capital costs, operational flexibility and steadiness, and environmental factors such as healthy water ways and lower greenhouse gas emissions.

In planning for the system operation, Melbourne Water prepares an annual operating plan (AOP) that provides a forecast of anticipated system operation for the water supply system. The AOP outlines the system operations for the 12 months ahead, and identifies for each month the volumes of water to be sourced, stored, moved from one location to another, or released to waterways. The preparation of this forecast is a complex task, as the decisions on system operations need to be made based on the array of considerations outlined earlier. In the past, the preparation of AOP was a manual process undertaken by experienced operators assisted by decision support tools based around the calculation of water balance across the system. However, these tools did not have the capability to identify optimal operations. Instead, the tools were manually updated by experienced operators as documented in system operation guidelines, in consultation with Melbourne's retail water companies. The above approach was time consuming, often requiring up to two weeks to produce an AOP for one operating scenario.

Although many researchers have attempted to develop methods to optimise the operation planning problem outlined above, it still remains largely a challenging research topic. This is partly due to the complexity of the problem involving multiple objectives and stakeholders, nonlinearities, uncertainties, complexity of water supply systems and the potentially long opimisation time horizon. Some of the research efforts that included the detailed optimisation of short-term or real-time operations, in addition to the long-term-oriented operating policy design, are as follows: Becker and Yeh (1974) developed a methodology utilising dynamic programming (DP) for the selection of an optimal reservoir storage policy over a specified number of policy periods, and linear programming (LP) for period-by-period optimisation. Gilbert and Shane (1982) presented three optimisation methods used for scheduling of a reservoir system that posed a stochastic, nonlinear, multireservoir problem. The methods included linear programming for implementing priorities, a nonlinear search method for minimising the cost function and a guide-development program based on stochastic dynamic programming. McLaughlin and Velasco (1990) described a real-time optimal control approach for operating two hydropower reservoirs. The approach included solving a simplified stochastic problem formulation of which the solution is obtained using a deterministic approach. Soncini-Sessa et al. (1999) presented a decision support system designed to be used to generate water reservoir management policies as well as to make daily operation decisions using real-time information. Castelleti et al. (2008) reviewed, from a control theory perspective, the advances in designing management policies for water reservoir networks and indicated that stochastic dynamic programming is the more natural algorithm for solving the problem of designing management policies for water reservoir networks. Simonović (2008) indicates that the most widely used optimisation methodology for water resource management has been LP. This is likely to be due its advantages including the wide availability of software and the ability to handle relatively large problems, despite the difficulties in representing non-linearities and uncertainties. Loucks et al. (2005) provides a thorough introduction to many aspects and dimensions of water resources management and presents practical approaches, including systems modeling and optimisation. Despite the above efforts, optimisation tools are not widely available or used in water resources management, particularly for large-scale problems.

OPTIMISIR is an easy-to-use optimisation tool, developed in-house by Melbourne Water, to assist with the preparation of AOP. The paper presents the OPTIMISIR modelling approach and an example application. The optimisation problem addressed by OPTIMISIR is one in which the operating decisions are interactively optimised over a period of one year, taking into account many operating considerations in detail. OPTIMISIR enables operations staff to investigate many operating strategies in a short period of time. However it does not address the optimisation of long-term operations or water resources requirements that is addressed by Optimizer WSS (Kularathna et al., 2011) which can provide some key inputs to OPTIMISIR.

The remainder of the paper is structured as follows: Section 2 outlines the Melbourne water supply system. Section 3 describes the model formulation and modeling process. An example application is presented in Section 4 , followed by summary and conclusions. 
Kularathna et al., Interactive Optimisation Modelling using OPTIMISIR to Support Melbourne Water Supply System Operation

\section{MELBOURNE WATER SUPPLY SYSTEM}

Melbourne Water is a Victorian government owned company that provides wholesale water supply and sewerage services to Melbourne's retail water companies, and manages rivers and creeks, floodplains and the regional drainage system. In addition, Melbourne Water provides limited water supplies to several regional water authorities. The water supply system managed by Melbourne Water is a complex interconnected system of 10 storage reservoirs, over 40 service reservoirs, 160,000 hectares of catchments and a transfer system comprising hundreds of kilometres of pipelines, tunnels and aqueducts. The transfer system links Melbourne's storage reservoirs with the areas serviced by the three retail water companies in Melbourne and regional water authorities. Figure 1 shows the water supply system managed by Melbourne Water on behalf of the above companies and water authorities who hold Melbourne's Bulk Water Entitlements.

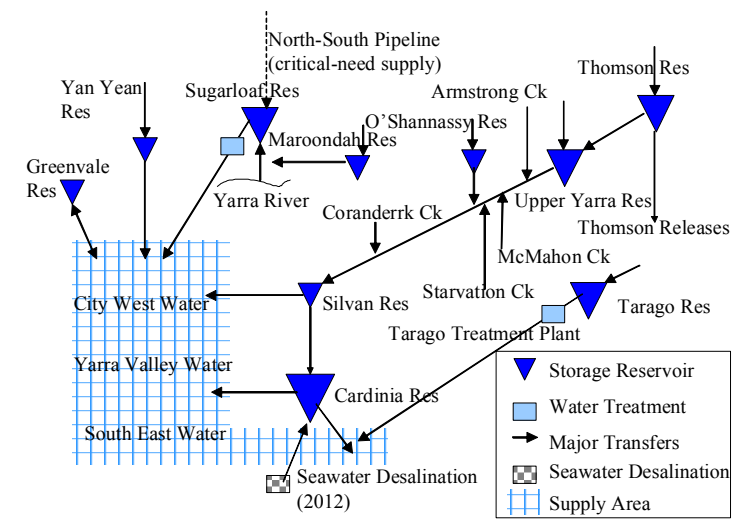

Figure 1. Melbourne water supply system.

Melbourne's ten storage reservoirs have a combined

storage capacity of 1,810 GL. The average volume of water supplied from the system for urban use over the last 5 years was about $390 \mathrm{GL} / y e a r$. The current water harvesting and transfer system comprises gravity fed supplies from protected catchments as well as fully treated and pumped supplies from unprotected catchments. The existing and new supply sources that involve water treatment and pumping can be of higher cost. However, some of these sources, such as the desalinated supply can be largely unaffected by climate.

\section{OPTIMISIR MODELLING APPROACH}

\subsection{Overview}

OPTIMISIR enables modelling of the optimal operation of Melbourne's water supply headworks system comprising water harvesting sites, storage reservoirs and bulk transfer paths over a period of 12 consecutive months. OPTIMISIR provides the flexibility for users to specify various inputs including the objective of the optimisation, starting system storage, streamflow and demand scenario, asset capacities, any operating requirements that must be met, environmental flow requirements and demand restriction rules. The output of OPTIMISIR comprises the optimal operating decisions for each of the 12 months for all key system components represented in the model, including: (1) volumes to be taken from various water sources, (2) volumes to be released to waterways at various water harvesting sites, (3) volumes to be stored in each reservoir, and (4) volumes to be transferred across various transfer paths in the system.

OPTIMISIR has two modes of use: (1) as a standalone optimisation tool to assist preparing the AOP, and (2) as an add-on to the existing water supply headworks system simulation model, REALM, to improve its simulation outcomes. The present paper covers the standalone mode. The second mode improves REALM simulation outcomes by representing optimal annual operation planning within a simulation model.

OPTIMISIR provides the choice of two optimisation methods: Linear Programming (LP) or Quadratic Programming $(\mathrm{QP})$, together with the flexibility for the user to specify the optimisation problem through input data. When using LP, the model developed for the Melbourne water supply headworks system representing 10 storage reservoirs and the bulk water transfer system runs in a couple of seconds in performing an optimisation for 12 months on a standard desktop computer used at Melbourne Water.

The model architecture of OPTIMISIR is shown in Figure 2 and comprises a Microsoft Excel user interface which 'communicates' with a standalone optimisation module through input and output files. The user interface provides a high level of flexibility to modify user input and output screens without requiring changes to the optimisation module.

\subsection{Model Formulation}

An optimisation model formulation comprises three key components; (1) objective function, OF, that define the aim of optimisation, (2) decisions that can be varied to achieve the objective, and (3) constraints that 
Kularathna et al., Interactive Optimisation Modelling using OPTIMISIR to Support Melbourne Water Supply System Operation

indicate the requirements that must be met or the limitations imposed on the optimisation. OPTIMISIR provides the flexibility to define all of these components through user inputs.

OPTIMISIR performs a single objective optimisation, which means that only one objective function can be optimised at a time. There are two objective functions preconfigured within OPTIMISIR for optimisation using LP. They are: (OF1) minimise the total annual cost in operating the system, and (OF2) minimise the total outflow from the system including localised spills from individual harvesting sites.

In using OF1, OPTIMISIR provides the option to account for the value of water held in storage, through an optional 'carryover water value function (CWVF)' (Kularathna, 2009) which can indicate how the value of water changes with the volume of water in storage. Such value functions need to be identified through economic assessments or long-term optimisation approaches. CWVF is one way of providing a link between the shortterm considerations of OPTIMISIR and the longer-term goals of system operation. In using OPTIMISIR for annual operation planning purposes, the above link may be provided through alternative ways. These include the incorporation of operation targets or pre-defining of some decisions based on longer-term optimisation processes and/or past experiences. The mathematical formulation of the model is included below with reference to the example system configuration shown in

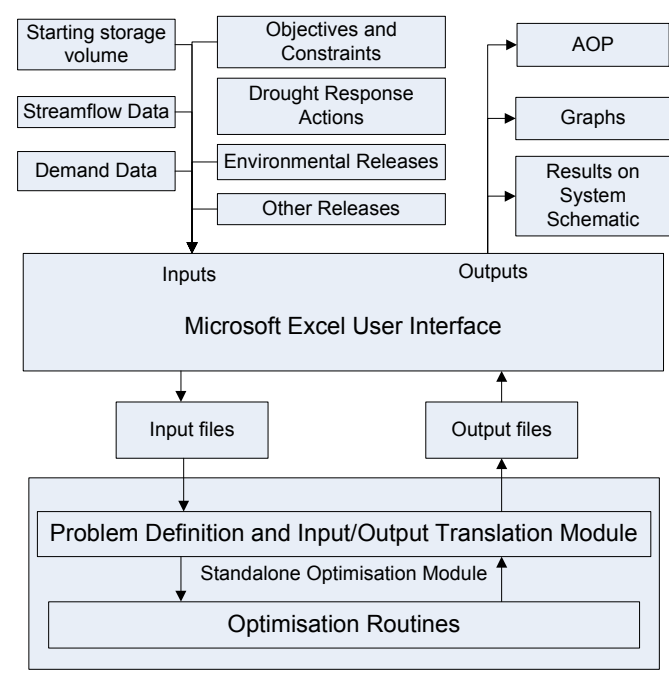

Figure 2. OPTIMISIR model architecture. Figure 3. The objective function OF1 has the form:

Minimise $\left[\sum_{t=1}^{12} \sum_{i=1}^{4} c_{i, t} \times o_{i, t}\right]-C W V F_{t=13}\left(s_{1, t}+s_{2, t}\right)$

where $c_{i, t}$ is the unit cost of water taken from location $i(i=1, . .4)$ in month $t, o_{i, t}$ is the volume taken from location $i$ in month $t$, and $C W V F_{t=13}$ is the optional function that indicates the value of the total volume of water available in storage for carryover to the $13^{\text {th }}$ month. $S_{i, t}(i=1,2)$ is the storage volume in reservoir $\mathrm{i}$ at the beginning of month $\mathrm{t}$.

The second objective function, OF2, has been constructed as the weighted sum of outflows and spills, with higher weights for outflows/spills from lower cost sources and vice versa. This provides OPTIMISIR with an approximate representation of the costs of various sources, within an objective function that minimises the

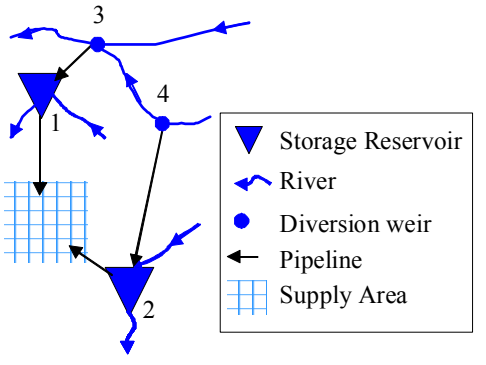

Figure 3. Example system. outflows. The objective function OF2 has the form:

Minimise $\sum_{t=1}^{12} \sum_{i=1}^{4} w_{i, t} \times r_{i, t}$

where $w_{i, t}$ is the weight applied to the downstream release volume $r_{i, t}$ of location $i$ in month $t$.

The key decisions of the optimisation problem are the outputs listed under Section 3.1. The constraints of the optimisation problem specify the 'water balance' at each modelled location, environmental flow requirements, water supply asset capacity limitations such as reservoir storage capacities and pipeline capacities, and, any operating conditions that must be satisfied.

The key constraints of the optimisation problem are of the general form outlined below.

$$
\begin{array}{ll}
s_{1, t+1}=s_{1, t}+q_{1, t}+o_{3, t}-o_{1, t}-r_{1, t}-e_{1, t} & t=1, . .12 \\
s_{2, t+1}=s_{2, t}+q_{2, t}+o_{4, t}-o_{2, t}-r_{2, t}-e_{2, t} & t=1, . .12
\end{array}
$$


Kularathna et al., Interactive Optimisation Modelling using OPTIMISIR to Support Melbourne Water Supply System Operation

$$
\begin{array}{ll}
q_{i, t}=o_{i, t}+r_{i, t} & i=3,4 ; t=1, . .12 \\
d_{t}=o_{1, t}+o_{2, t} & t=1, . .12 \\
r_{i, t} \geq r_{i, t}^{\min } & i=1, . .4 ; t=1, . .12 \\
s_{i, t}^{\min } \leq s_{i, t} \leq s_{i, t}^{\max } & i=1,2 ; t=1, . .12 \\
o_{i, t}^{\min } \leq o_{i, t} \leq o_{i, t}^{\max } & i=1, .4 ; t=1, . .12
\end{array}
$$

where $q_{i, t}$ is the inflow in month $t$ of the catchment between the location $i$ and any upstream locations modelled, $e_{i, t}(\mathrm{i}=1,2)$ is the volume lost from location $i$ in month $t, d_{t}$ is the water demand from the system in month $t, r_{\mathrm{i}, \mathrm{t}}{ }^{\text {in }}$ is the minimum required flow downstream of location $i$ in month $t, s_{\mathrm{i}, \mathrm{t}}{ }^{\max }$ and $\mathrm{s}_{\mathrm{i}, \mathrm{t}}{ }^{\text {min }}$ are the maximum and minimum allowable storage volumes in reservoir $i$ in month $t, \mathrm{O}_{\mathrm{i}, \mathrm{t}}{ }^{\text {max }}$ and $\mathrm{o}_{\mathrm{i}, \mathrm{t}}$, maximum and minimum volumes that can be transferred out of location $i$ in month $t$.

The optimisation routines used in OPTIMISIR were sourced from Visual Numerics (2006). The LP routine is based on revised simplex method of which a description can be found in Murty (1983). The QP routine is based on the implementation of Powel (1985) of the dual quadratic programming algorithm of Goldfarb and Idnani (1983) for convex QP problems subject to general linear equality / inequality constraints. The LP routine solves a problem with a linear objective function and constraints, whereas the QP routine provides the flexibility of using a quadratic objective function. In using the OF1, the optional and potentially non-linear CWVF was represented through a piecewise linearisation approach.

\subsection{Typical Modelling Process}

OPTIMISIR has no built-in operating rules and can identify at first a 'greenfield solution' which is not biased towards past operations. A greenfield solution may show the best way to optimise the system under ideal conditions. However, such greenfield solutions may not satisfy all operating considerations associated with a complex water supply system. Hence, OPTIMISIR modeling process involves subsequent incremental adjustments to the greenfield model formulation to identify revised optimal solutions ('constrained solutions') that satisfy various operating requirements. The incremental adjustments enable users to identify any system-wide implications of each specific adjustment, prior to making further adjustments.

\section{EXAMPLE APPLICATION}

The example application is intended to illustrate: (1) the optimisaton capabilities of OPTIMISIR, and (2) the modelling adjustments indicated in Section 3.3 and does not indicate the potential operation of Melbourne water supply system. Description of the assumptions used in the example including streamflow scenarios has been intentionally omitted from this paper to reflect the above intent.

OPTIMISIR was run for three separate streamflow scenarios using OF2. The three scenarios are named as 'drier', 'intermediate' and 'wetter' scenarios for ease of reference, although the names do not imply the level of streamflow in relation to the whole range of possible streamflow conditions. The constraints of the model formulation specified environmental flow requirements and water supply asset capacity limitations. Figure 4 presents the modelled total system storage volumes corresponding to the greenfield solutions obtained using the three scenarios. In Figure 4, the dashed line represents the operating plan for the intermediate streamflow scenario, prepared through the manual process referred to in Section 1. Comparison of the outcomes of the manual process and OPTIMISIR indicate the closeness of the outcomes of the two approaches in terms of the total system storage volume. In terms of the estimated operating cost, the two solutions were identical. This result highlights the optimality achieved in the past operation planning

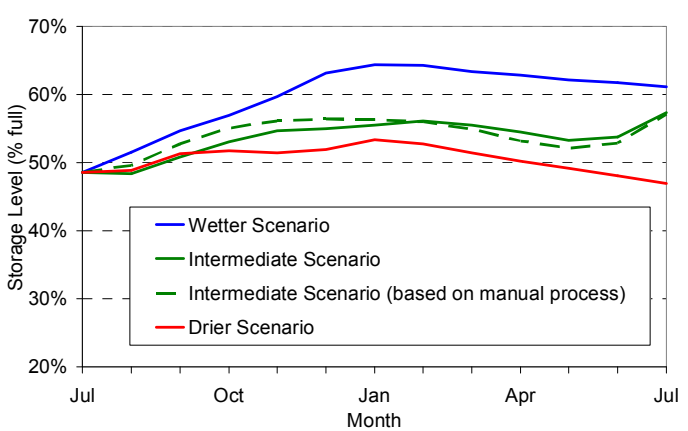

Figure 4. Modelled storage volume. process that relied on operating guidelines, past experience and the expertise of operators. 

System Operation

Figure 5 indicates the normalised operating cost and system outflow associated with the three streamflow scenarios. In Figure 5, the optimal cost under the drier scenario is slightly lower than that under the intermediate scenario. This is because the drier scenario represents limited availability of relatively low-cost surface water sources, and the maximisation of higher cost sources. The wetter scenario has the lowest cost due to the availability of a large volume of low-cost surface water and consequent reduction of intake from higher cost sources. The intermediate scenario has the highest cost as the available low-cost source volume is not large enough to cause a reduction in the intake of higher cost sources.

In terms of outflow volumes, the drier scenario has the highest outflow from the system, because of the larger volume that needs to be released from the system to ensure environmental flow requirements are met, when the natural streamflow downstream is relatively low. Conversely, the wetter scenario has the lowest outflow, not only because the streamflow downstream is relatively high but also because the optimal solution avoided any potential localised spills.

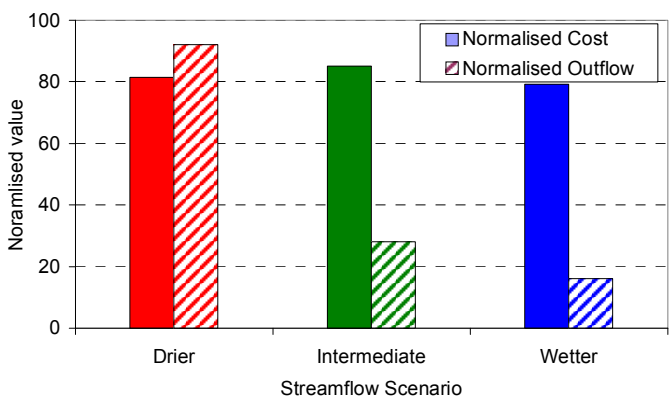

Figure 5. Modelled cost and outflow.

Although OPTIMISIR is a single objective optimisation tool, it can also be used to: (1) check the optimality of a solution in regards to another objective, (2) generate alternative solutions that are optimal in terms of other objectives, and (3) to identify optimal solutions that trade-off different objectives to different extents. Such alternative solutions can be generated by revising the optimisation model formulation interactively. As an example, the solution obtained for the intermediate scenario using OF2 (which causes the end-storage volume to be maximised) was tested by optimising the system under OF1, with the end-storage volume constrained to be no less than that identified using OF2. The two solutions were identical, indicating that for this particular set of start storage, streamflow scenario and constraints, the end-storage volume has been maximised at the lowest cost. This is partly because the OF2 also has an approximate representation of costs, specified by the relative weights on outflows/spills from different sources.

When using OF1, OPTIMISIR can be used to identify optimal solutions that trade-off different objectives to different extents by gradually varying the end-storage constraint to generate solutions that have different end-storage volumes and costs. A similar outcome can be obtained using OF2 with different constraints on operating costs. The solutions generated using such an approach are equally good and optimal solutions, known as Pareto-Optimal solutions (or Pareto Front), from which the selection of a solution involves a trade-off such as cost versus end-storage volume. An alternative method for generating a Pareto Front is to minimise the weighted sum of the different objectives for various different settings of the weights. Although OPTIMISIR provides the flexibility to use the weighted sum approach, the single objective function approach, with constraints on end-storage volume or cost, can represent the practical considerations in a more transparent manner. This approach also enables focusing on the areas of the Pareto Front that are of practical interest and applicability, without having to generate the entire Pareto Front.

Figure 6 presents the Pareto Front generated for the intermediate streamflow scenario using $\mathrm{OF} 2$ and different operating-cost constraints. The solutions $\mathrm{A}$ and B shown in Figure 6 are the best in terms of cost and end-storage respectively. The solutions inbetween represent a trade-off cost and end-storage to different extents. The lower-cost end of Pareto Front indicates the use of lower-cost sources. The highercost end indicates that further relaxation of operating cost-constraint has no impact on end-storage, due to limitations in water availability and/or localised capacity constraints. The intermediate linear section indicates the increasing use of a higher-cost source which was assumed to be of a constant unit cost.

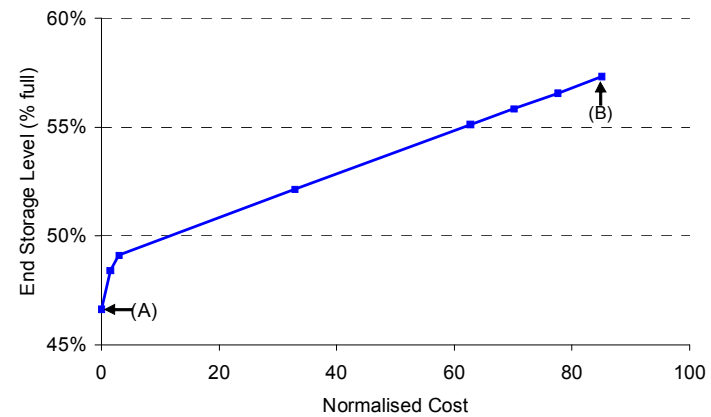

Figure 6. Pareto-Optimal solutions.

Figure 7 provides an example containing greater details of a greenfield solution and the subsequent constrained solution, for two reservoirs in the system. Figure 7(a) indicates the outcome of adjustments made to the greenfield formulation to ensure that the storage volume of Maroondah reservoir is not drawn down excessively in anticipation of higher streamflows, and, a sufficient empty storage space is maintained at the 
Kularathna et al., Interactive Optimisation Modelling using OPTIMISIR to Support Melbourne Water Supply System Operation
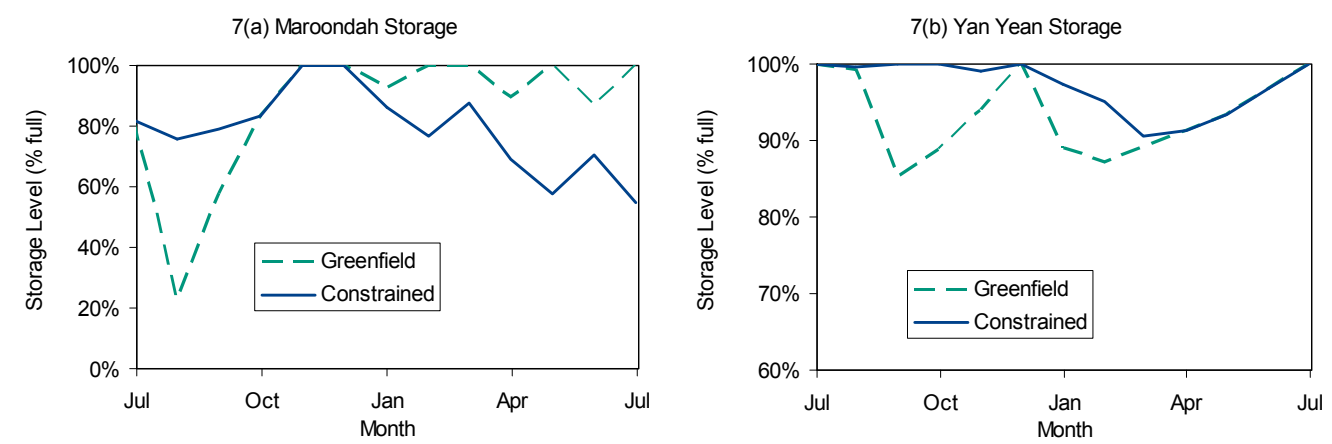

Figure 7. Details of greenfield and constrained solutions.

end of the 12 months to maximise the potential intake next year. Figure 7(b) indicates the outcome of adjustments made to achieve stable operation of Yan Yean reservoir.

\section{SUMMARY AND CONCLUSIONS}

The paper described an interactive water supply system optimisation tool, OPTIMISIR, developed to assist with the preparation of annual operating plan for the Melbourne water supply system. OPTIMISIR provides the flexibility for the user to generate an optimal operating plan by interactively defining the optimisation problem through objectives, constraints and various input data. OPTIMISIR modeling process involves first identifying an optimal 'greenfield solution' which is not biased towards past operations. Any specific operating considerations not represented in the greenfield solution can subsequently be incorporated to generate an optimal 'constrained solution' that best satisfies the specific considerations. The outputs of the tool provide valuable insights into the system-wide implications of various operating considerations. The ability to generate an optimal solution within a couple of seconds is a key advantage of the tool. Recent use of OPTIMISIR has shown its potential, as part of a suite of tools, for efficiency improvements in annual operating plan preparation. The capabilities of OPTIMISIR also highlight its potential as a training tool that demonstrates optimal system operations and the implications of various operating considerations.

\section{REFERENCES}

Becker, L., and W.W-G. Yeh (1974). Optimization of real time operation of a multiple-reservoir system. Water Resources Research, 10(6), 1107-1112.

Castelletti, A., F. Pianosi and R. Soncini-Sessa (2008). Water reservoir control under economic, social and environmental constraints. Automatica, 44, 1595-1607.

Gilbert, K.C., and R.M. Shane (1982). TVA Hydro Scheduling Model: Theoretical Aspects. Journal of the Water Resources Planning and Management Division, 108(1), 21-36.

Goldfarb, D., and A. Idnani (1983). A numerically stable dual method for solving strictly convex quadratic programs. Mathematical Programming, 27, 1-33.

Kularathna, M.D.U.P. (2009). OPTIMISIR: An optimisation tool to supplement REALM models or perform standalone optimisation of water resource systems. 18th World IMACS / MODSIM Congress, Cairns, Australia, 13-17 July.

Kularathna, M.D.U.P., T.S.C. Rowan, H. Schultz-Byard, D.R. Broad, D. McIver, D. Flower, B. Baker, B. Rhodes and P.J. Smith (2011). Multi-Objective optimisation using Optimizer WSS to support operation and planning decisions of Melbourne water supply system. Submitted to MODSIM 2011 International Congress on Modelling and Simulation, Perth, Australia, 12-16 December.

Loucks, D.P., and E. van Beek (2005). Water Resources Systems Planning and Management: An Introduction to Methods, Models and Applications. Studies and Reports in Hydrology, UNESCO, Paris.

McLaughlin, D., and H.L. Velasco (1990). Real-time control of a system of large hydropower reservoirs. Water Resources Research, 26(4), 623-635.

Murty, K. G. (1983). Linear Programming. John Wiley and Sons, New York.

Powell, M.J.D. (1985). On the quadratic programming algorithm of Goldfarb and Idnani. Mathematical Programming Study, 25, 46-61.

Simonović, S.P. (2008). Managing water resources: methods and tools for a systems approach. Studies and Reports in Hydrology, UNESCO / Earthscan, London, 429-440.

Soncini-Sessa, R., A.E. Rizzoli, L. Villa, and E. Weber (1999). TwoLe: A software tool for planning and management of water reservoir networks. Hydrological Science Journal, 44(4), 619-631.

Visual Numerics (2006). IMSL Fortran numerical library user's guide. 